\title{
Intelligent Fault Diagnosis of Rotating Machine Based on SVMs and EMD Method
}

\author{
Zhengkai Zhang ${ }^{1, *}$, Lichen $\mathrm{Gu}^{1}$ and Yongsheng Zhu ${ }^{2}$
}

\author{
${ }^{I}$ School of Mechanical \& Electrical Engineering, Xi'an University of Architecture and Technology, Xi'an City, PR \\ China: ${ }^{2}$ Key Laboratory of Education Ministry for Modern Design and Rotor-Bearing System, Xi'an Jiao tong Univer- \\ sity, Xi'an City, PR China
}

\begin{abstract}
Empirical mode decomposition (EMD) is a self-adaptive analysis method for signal process. Because the EMD method is highly efficient in non-stationary and nonlinear data analysis. It has been widely applied to fault diagnosis of rotating machine. However, EMD method is not suitable for the Intelligent fault diagnosis, because the number of intrinsic mode functions (IMFs) is unfixed. In this paper, a classification method based on correlation coefficient was present, which can establish a one-on-one relationship between IMFs which decomposed from different signals by EMD method. And then, the feature of each IMFs is extracted and evaluated by using Support vector machines (SVMs). That will make the intelligent fault diagnosis possible. In order to prove the effectiveness of the method, the proposed method is applied to fault diagnosis on the signals get from a test rig.
\end{abstract}

Keywords: Empirical mode decomposition, Intrinsic mode function, correlation coefficient, support vector machines.

\section{INTRODUCTION}

With rapid development of scientific technology, mechanical equipment in modern industry is growing more automatic and more sophisticated. So Intelligent fault diagnosis plays a more and more important role in modern industry.

Intelligent fault diagnosis usually include two aspects. The first, the specific parameters which can characterize the mechanical status are extracted from the measured signal. Then the modern artificial intelligence methods (such as neural networks, support vector machines, etc) are introduced on the characteristics classification and identification.

According to existing research, although some mechanical equipment failure is very complex, but still has its own pattern, and these pattern can be described by "frequency channel" or "time-frequency channel". In other words, the fault occur and develop through the "channel". For example, rotor imbalance fault, activity channel is rotating frequency. When the imbalance is weak, the energy pass by the rotating frequency channel is limited, when the imbalance is serious, then the energy pass by rotating frequency channel is large. For rotor misalignment fault, activity channel is rotating frequency and its octave. For more complex fault such as rubimpact fault, its pattern also can be described by a specific "frequency channel" in frequency domain or "time-frequency channel" in time-frequency domain. When the rotor is running at different status, the energy passing by a particular channel is different, and some channels are closed. If we can identify the fault-related "frequency channel" or "time-

*Address correspondence to this author at the School of Mechanical \& Electrical Engineering, Xi'an University of Architecture and Technology, Xi'an City, PR China; Email: woodncy@163.com frequency channel", then we can get a comprehensive evaluation of the entire mechanical equipment by the amount of energy pass through various channels.

In order to realize the above ideas, we need a selfadaptive decomposition method which can decompose the signal in accordance with the frequency band or timefrequency band. So we use EMD method to solve this problem.

According to existing research, the EMD method is an effective self-adaptive dyadic filter bank [1], and has a series of advantages over other signal analysis methods for its ability of handling nonlinear and non-stationary signals. Because EMD method is a self-adaptive analysis method which can decompose a complicated signal into a collection of intrinsic mode functions (IMFs) based on the local characteristic time scale of the signal and is particularly effective for the decomposition of nonlinear and non-stationary signal. It has been developed and widely applied in fault diagnosis of rotating machinery, for example, rolling bearing fault diagnosis [2-4], gear fault diagnosis [5], and rotor fault diagnosis $[6,7]$.

But as a relatively new signal processing methods, EMD method also not very mature and suffers from some unsolved deficiencies, which brought a number of problems in practice. Such as mode mixing [8] and the end swings near the ends of signal [9]. Moreover, EMD method is not suitable for intelligent fault diagnosis. Because the EMD method is a self-adaptive analysis method, the number of IMFs which decomposed from a signal is determined by the complexity of the signal. The IMFs represent the natural oscillatory mode embedded in the signal and work as the basis functions, which are determined by the signal itself, rather than pre-determined kernels [8]. Under such conditions, if the criterion to terminate the calculation are the same, then the 
number of IMFs which decomposed from different signals may be different. For example, two simulative signals:

$$
\begin{aligned}
& x(t)=\sin (2 \pi \times 10 t+\pi) \sin (2 \pi \times 0.1 t)+20 \sin (2 \pi \times 0.3 t) \\
& y(t)=3 \sin (2 \pi \times 10 t) \sin (2 \pi \times 0.1 t)+12 \sin (2 \pi \times 0.3 t)+3 \sin (2 \pi \times 2 t)
\end{aligned}
$$

All the IMFs of $x(t)$ and $y(t)$ have been shown in Fig. (1a), and (1b), respectively.

From Fig. (1), we can find that, although the amplitude is different, the $\mathrm{IMF}_{\mathrm{x} 1}$ in Fig. (1a) and $\mathrm{IMF}_{\mathrm{y} 1}$ in Fig. (1b) illustrate the same frequency intrinsic mode, and the $\mathrm{IMF}_{\mathrm{x} 2}$ in Fig. (1a) and IMF $\mathrm{IM}_{\mathrm{y}}$ in Fig. (1b) illustrate the same frequency intrinsic mode. So, these IMFs which have the same frequency characteristics should be placed together to observe and compare in signal analysis.

- But if the two series of IMFs are compared one-on-one in accordance with the order, the two IMFs contain the same physical meaning will not be able to compared, and naturally can not get the correct results.

- From the principle of EMD method, each IMFs should have a clear physical meaning. Only when we know the precise physical meaning of each IMFs, can we put the IMFs which decomposed from different signal together for comparison. When the EMD method is used, a signal processing expert is required to complete the abovementioned work, so it is difficult to use EMD method for intelligent fault diagnosis.

- To alleviate the above-mentioned problem. An improved method is presented in this article to assess the degree of similarity of two IMFs, and the IMFs which decomposed from signal are reordered in accordance with this approach.

- Recently, fault diagnosis of mechanical equipment has widely used artificial intelligence methods. Such as artificial neural networks and support vector machines
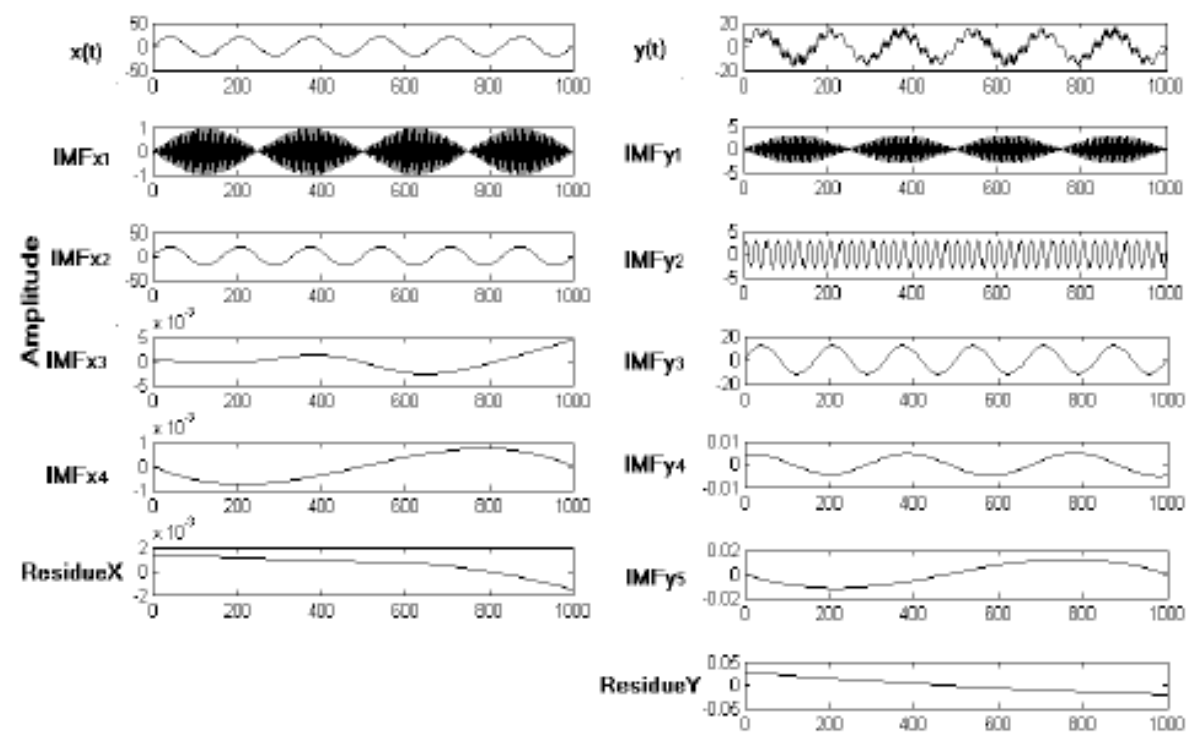

(a)
(SVMs) have been successfully used in practical applications [10-12]. SVMs is a universal learning algorithm, which implement the structure risk minimisation (SRM) principle proposed by statistical learning theory (STL), gradually become the hot research point in the field of artificial intelligence for its favorable generalisation ability. In this paper, the task of the intelligent identification and classification is completed by SVMs.

- This paper is organised as follows. Section 2 gives a brief review of the EMD method and SVMs. In Section 3, the pre-processing on IMFs which decomposed from two simulative signals $x(t)$ and $y(t)$ based on correlation coefficient are introduced. The experiments and data sets are introduced in Section 4. Section 5 discussed the classification performance. The conclusion of this paper is given in Section 6.

\section{A BRIEF REVIEW OF EMD METHOD AND SVMS}

\subsection{EMD Algorithm}

EMD method is developed from the simple assumption that any signal consists of different simple intrinsic modes of oscillations. Each linear or non-linear mode will have the same number of extrema and zero-crossings. There is only one extremum between successive zero-crossings. Each mode should be independent of the others. In this way, each signal could be decomposed into a number of intrinsic mode functions (IMFs), each of which must satisfy the following definition [13]:

(1) In the whole data set, the number of extrema and the number of zero crossings must either equal or differ at most by one.

(2) At any point, the mean value of the envelope defined by local maxima and the envelope defined by the local minima is zero.

(b)

Fig. (1). IMFs of two different signal:(a) $x(t)$ and (b) $y(\mathrm{t})$. 
The IMFs indicate the simple oscillation mode involved in the signal. EMD, a "sifting" process, is used to extract the IMFs by the following steps [13]:

(1) Identify all the extrema of the signal, and connect all the local maxima by a cubic spline line as the upper envelope. Repeat the procedure on the local minima to produce the lower envelope.

(2) Designate the mean of the two envelopes as $m_{1}$, and the difference between the signals $\mathrm{s}(\mathrm{t})$ and $\mathrm{m}_{1}$ as the first component, $h_{1}$, i.e.

$\mathrm{s}(t)-m_{1}(t)=h_{1}(t)$.

If $h_{1}$ is an IMF, take it as the first IMF of $x(t)$. If $h_{1}$ is not an IMF, take it as the original signal and repeat the steps above until $h_{1 k}$ is an IMF, and designate $h_{1 k}(t)$ as $c_{1}(t)$ :

$c_{1}(t)=h_{1 k}(t)$.

(3) Separate the first IMF $c_{1}(t)$ from $x(t)$ by

$\mathrm{s}(t)-c_{1}(t)=r_{1}(t)$.

(4) Treat residue $r_{1}(t)$ as the original signal and subject it to the same process as above, so that we can get other IMFs, $c_{2}, c_{3}, \ldots, c_{\mathrm{n}}$, which satisfy

$\left.\begin{array}{c}r_{1}(t)-c_{2}(t)=r_{2}(t) \\ \cdot \\ \cdot \\ r_{n-1}(t)-c_{n}(t)=r_{n}(t)\end{array}\right\}$

(5) By summing up Eq. (5) and Eq. (6), we finally obtain:

$\mathrm{s}(t)=\sum_{i=1}^{n} c_{i}(t)+r_{n}(t)$

Thus, one can achieve a decomposition of the signal into n-empirical modes, and a residue $r_{n}(t)$, which is the mean trend of $s(t)$. The IMFs $c_{1}(t), c_{2}(t), \ldots, c_{\mathrm{n}}(t)$ include different frequency bands ranging from high to low. The frequency components contained in each frequency band are different and change with the variation of signal $s(t)$. So, EMD is a self-adaptive signal decomposition method.

\subsection{Support Vector Machines}

SVMs is a learning algorithm, which come from an optimal separating hyper-plane in case of linearly separable, were developed by Vapnik and his co-workers [14]. Its core idea is to map the original pattern space into the high dimensional feature space $\mathrm{Z}$ through some non-linear mapping functions, and then construct the optimal separating hyperplane in the feature space. Thus, the non-linear problem in low dimensional space corresponds to the linear problem in the high dimensional space [15].

The input vector $\mathrm{v}$ is mapped into the high dimensional space $\mathrm{Z}$ through the non-linear mapping function $\varphi(v)$, and the linear function sets

$$
f(\mathrm{v}, \alpha)=(\omega \cdot \varphi(v))+b
$$

The corresponding constraint optimisation problem is

$$
\begin{array}{ll}
\min & \frac{1}{2}\|\omega\|^{2}+C \sum_{i=1}^{l} \xi_{i} \\
\text { s.t. } & \begin{cases}\mathrm{w}_{i}\left(\omega \cdot \varphi\left(\mathrm{v}_{i}\right)\right)+b \geq 1-\xi_{i}, & i=1, \ldots, l \\
\xi_{i} \geq 0, & , i=1, \ldots, l\end{cases}
\end{array}
$$

where the coefficient $C$ is a penalty factor, and it implements a trade-off between empirical risk and confidence interval. The coefficient $\xi_{i}$ is a slack factor.

Eq. (9) is a classical convex optimisation problem. The calculation can be simplified by converting the problem with Kuhn-Tucker condition into the equivalent Lagrangian dual problem, which will be:

$$
\min L(\omega, b, \alpha)=\frac{1}{2}\|\omega\|^{2}-\sum_{i=1}^{l} \alpha_{i} \mathrm{w}_{i}(\omega \cdot \varphi(v)+b)+\sum_{i=1}^{l} \alpha_{i} .
$$

The solution of Eq. (10) can be resolved by vanishing the derivation of $L$ with respect to o and the derivation of $L$ with respect to $\alpha$, and then the following equation can be obtained:

$$
\omega=\sum_{i=1}^{l} \alpha_{i} \mathrm{w}_{i} \varphi\left(\mathrm{v}_{i}\right), \quad \sum_{i=1}^{l} \alpha_{i} \mathrm{w}_{i}=0,
$$

where $\alpha_{i}$ is the Lagrangian multiplier. Combining Eqs. (20) and (11), we can get the dual quadratic optimization problem

$$
\begin{aligned}
& \max \quad L(\alpha)=\sum_{i=1}^{l} \alpha_{i}-\frac{1}{2} \sum_{i, j=1}^{l} \alpha_{i} \alpha_{j} \mathrm{w}_{i} \mathrm{w}_{j} \varphi\left(v_{i}\right) \cdot \varphi\left(v_{j}\right), \\
& \text { s.t. } \quad \alpha_{i} \geq 0, \quad \sum_{i=1}^{l} \alpha_{i} \mathrm{w}_{i}=0 .
\end{aligned}
$$

Thus, by solving the dual optimisation problem, one obtains the coefficient $\alpha_{i}$ which is required to express the $\omega$ to solve Eq. (13). This leads to non-linear decision function:

$$
f(x)=\operatorname{sign}\left(\sum_{i=1}^{l} \alpha_{i} \mathrm{w}_{i}\left(\varphi\left(v_{i}\right) \cdot \varphi\left(v_{j}\right)\right)+b\right) .
$$

In Eq. (14), the inner product $\varphi\left(v_{i}\right) \cdot \varphi\left(v_{j}\right)$ needs to be computed in the feature space. According to kernel function theory, we can use the kernel function $K\left(v_{i}, v_{j}\right)$ in the input space, which satisfies the Mercer condition, to compute the inner product. So Eq. (14) can be expressed as:

$$
f(x)=\operatorname{sign}\left(\sum_{i=1}^{l} \alpha_{i} \mathrm{w}_{i} K\left(v_{i}, v_{j}\right)+b\right) .
$$

The typical examples of kernel function are polynomial kernel, radial basis function (RBF) kernel, sigmoid kernel and linear kernel. In many practical applications, the RBF kernel obtains the highest classification accuracy rate than other kernel functions, so, in the present paper, we mainly consider the RBF kernel.

Support vector machines were originally designed for binary classification. Currently there are several methods that have been proposed for multi-class classification, such as "one-against-one", "one-against-all". In this study, we adopt "one-against-one" method [16] to identify the different faults. 


\section{THE PRE-PROCESSING ON IMFS}

\subsection{IMFs Selection}

From the EMD method, we can find that the IMFs should be a kind of complete, adaptive and almost orthogonal representation for the analysed signal under ideal circumstances. But in practice, there are always some undesirable IMFs generated during the process of EMD, especially in the lowfrequency components [9]. These undesirable IMFs are considered as pseudo-components of the signal, not only does not contain any useful information, but have an adverse impact on the signal analysis and identification. These pseudocomponents should be eliminated before the classification and sorting of the IMFs.

From [9] we can find that these pseudo-components are caused by end swing effect. The end swing effect is usually encountered in the analysis of a finite length signals. Using EMD method is destined to have pseudo-components.

However, we find that the amplitude of pseudocomponents is very sensitive to the boundary change of the original signal, this is a clear distinction with true IMFs.

In this paper, we will have two different EMD decomposition operation for the same original signal $y(t)$. In the first operation, $y(t)$ will be cut at both ends of $15 \%$, then the remainder will be decomposed by EMD method, we call the obtained IMF as IMF $\mathrm{Ie}_{\mathrm{ce}}$.

In the second operation, we will decomposed the original signal by EMD method at first, then the IMFs will be cut at both ends of $15 \%$, we call the obtained IMF as $\mathrm{IMF}_{\mathrm{ec}}$.

Through the EMD decomposition of $y(t)$, the $\mathrm{IMF}_{\text {ec }}$ and $\mathrm{IMF}_{\mathrm{ce}}$ are shown in Fig. (2a) and Fig. (2b) separately.

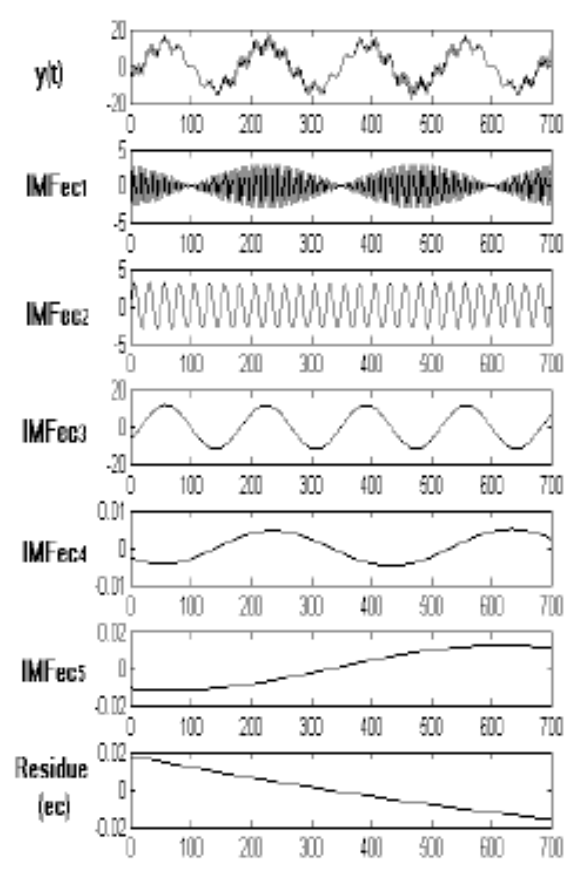

(a)
In this paper, we use correlation coefficients to determine similarity of two IMFs. The correlation coefficient is a quantity that gives the quality of a least squares fitting to the original data. The correlation coefficient is 1 in the case of the two IMFs are equal, -1 in the case of the two IMIFs have a opposite relationship, and some value in between in all other cases.

Theoretically speaking, the two groups of IMFs we obtain through two process are come from the same original signal. If the IMF is true component, then the correlation coefficient of two corresponding IMFs should be equal or very close to 1 . If the IMF is pseudo-component, then it is generated by end swing effect, because the end conditions in two process are different, the correlation coefficient of two corresponding IMFs should be smaller.

Through the comparison of Fig. (2a) and Fig. (2b), we can find that the first three IMFs of two groups are consistent on the amplitude and phase. So we consider them as true components, and the rest IMFs as pseudo-components. The correlation coefficients of corresponding $\mathrm{IMF}_{\mathrm{ec}}$ and $\mathrm{IMF}_{\mathrm{ce}}$ were shown in (Table 1).

In order that the computer can automatically remove these pseudo-components. We set a hard threshold $\lambda=0.9$, so that the computer can automatically remove these IMFs whose corresponding correlation coefficients are less than $\lambda$.

After IMFs selection, pseudo-components have been eliminated, and the residual IMFs are shown in (Fig. 3).

\subsection{Re-Sequencing of IMFs}

As mentioned earlier, EMD method is developed from the simple assumption that any signal consists of different

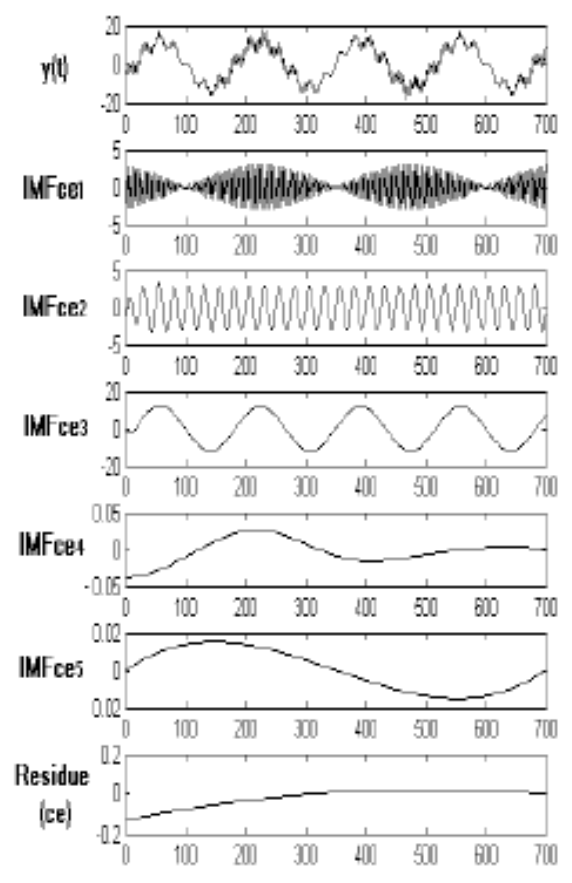

(b)

Fig. (2). (a). $I_{\mathrm{MF}}$ (b). $I M F_{c e}$ 
Table 1. The Correlation Coefficients of IMFec and IMFce

\begin{tabular}{|c|c|c|c|c|}
\hline & $\mathbf{1}$ & $\mathbf{2}$ & $\mathbf{3}$ & $\mathbf{4}$ \\
\hline \hline Corrcoelation coefficients & 0.999 & 0.992 & 0.999 & 0.816 \\
\hline
\end{tabular}

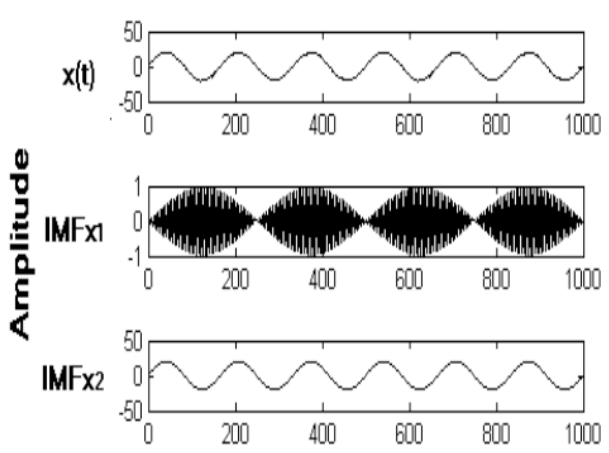

Fig. (3). Residual IMFs.

simple intrinsic modes of oscillations. This assumption is coincide with the practical situation in most cases.

Because the vibration frequency caused by a particular physical effects always confined to a specific frequency band, so the IMFs which decomposed from a signal obtained by actual measurement are also confined to a specific frequency band too, then from the spectrum can we judge the existence of similar relations between the IMFs, that is to say whether these IMFs are the result of the same intrinsic mode. However, the Fourier transform is not suitable for the frequency domain analysis of nonlinear signal, we have to use power spectral density (PSD) to analysis the frequency domain of the IMFs.

Power spectral density (PSD) is used to describe the power of a signal distributes with frequency. It is a statistical method generally used for random vibration analysis. In this paper, we use Burg method to make an estimate of the power spectral density (PSD) of the IMFs.

Burg's method has the particular advantage of always producing stable models and handling short data records well. More information about Burg's method can be found in [17]. The PSDs of each IMFs are shown in (Fig. 4).

From Fig. (5), we can find that the PSD of $\mathrm{IMF}_{x 1}$ are very similar to $\mathrm{IMF}_{y 1}$, the PSD of $\mathrm{IMF}_{x 2}$ and $\mathrm{IMF}_{y 3}$ are very similar, too.

In order to better understand their relationship, we put them on the same figure and the PSDs of these IMFs have been normalised at first so as to reduce the interfere of amplitude.

The similarity of PSD shows that these IMFs have the same power distribution in the frequency domain. So we can consider these IMFs are generated by the same type of vibration mode. In other words, these IMFs can be considered as

\section{$y(t)$}

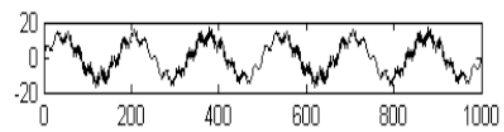

IMFy!

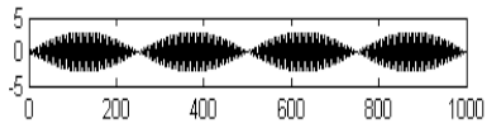

IMFy2

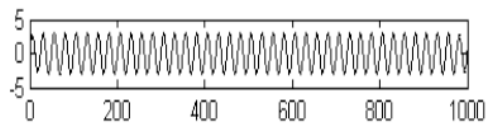

IMFy3

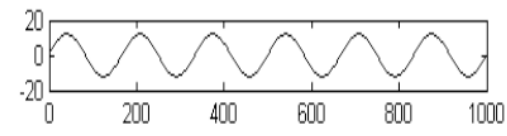

from the same 'channel'. If we can find the similar to the above one-on-one relationship among these IMFs which decomposed from different signal, then a effective and accurate comparison among these IMFs can be carried out.

In accordance with the requirements of intelligent diagnosis, the results of the comparison can not come from the subjective judgments of the operator, so we need a quantity to represent the similarity of different PSDs. In this paper, we have adopted the frequency-domain correlation coefficient $\left(\right.$ Cor $\left._{\text {Freq }}\right)$ to represent the similarity relationship between PSDs.

The correlation coefficient, sometimes also called the cross-correlation coefficient, is a quantity that gives the quality of a least squares fitting to the original data. Correlation coefficient indicates the strength and direction of a linear relationship between two random variables.

Correlation coefficient is often used in the correlation and similarity analysis of two time-domain signal, but the correlation coefficient has been used to analysis the similarity of optical spectrum [18].

In [18], a database was set up at first which contains the optical spectrum of various plants. Then, when the species of a plant need to be identified. An optical spectral analysis was carried out on this plant, and the spectral correlation analysis was carried out between the obtained optical spectrum and the optical spectrum stored in the database. When the spectral correlation coefficient between the unknown plant and a known plants stored in database is greater than a threshold, the unknown types of plant belongs to the corresponding plant species. In this way, the species of plants which contain complex composition can be determined automatically.

In this paper, we also adopted a similar approach. We set a hard threshold $\eta=0.5$, so that the computer can automatically determine the one-on-one relationship between IMFs. 

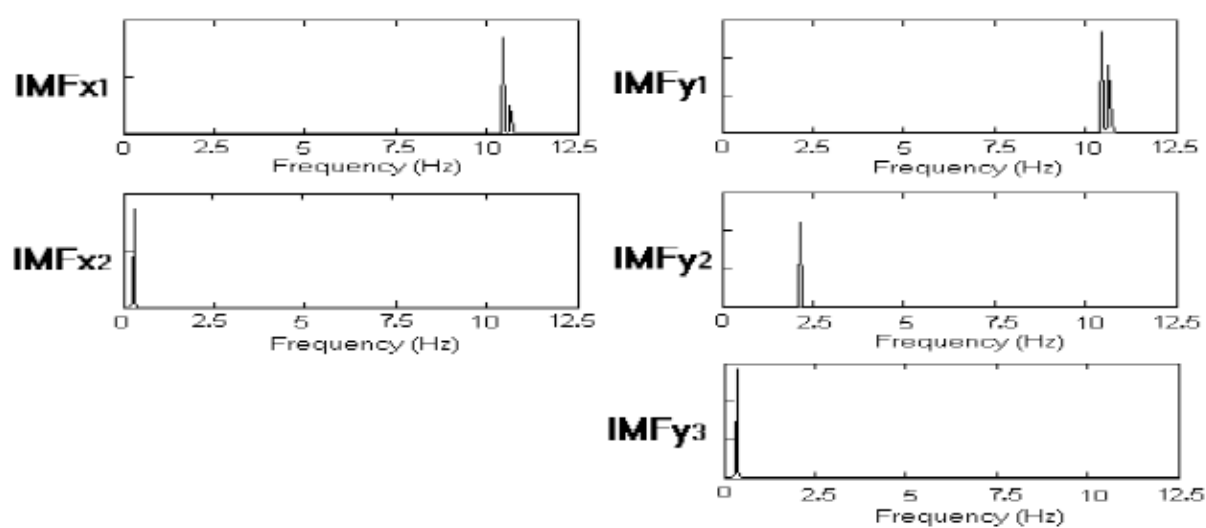

Fig. (4). PSDs of IMFs.

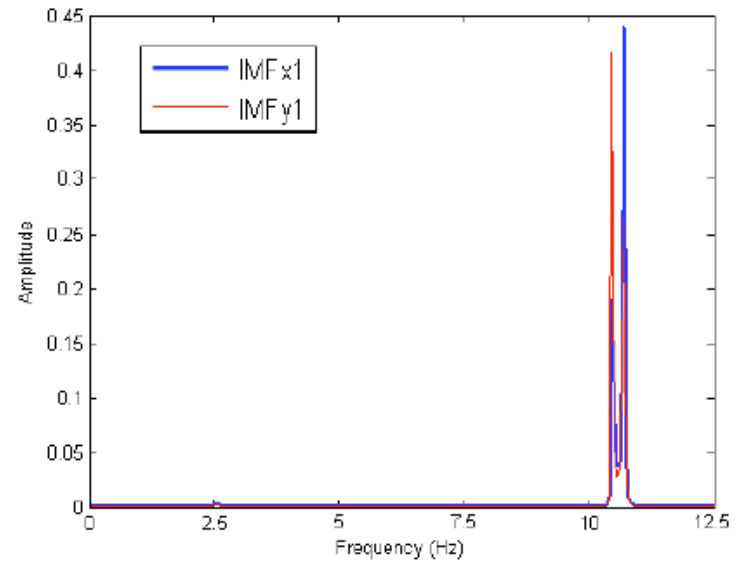

(a)

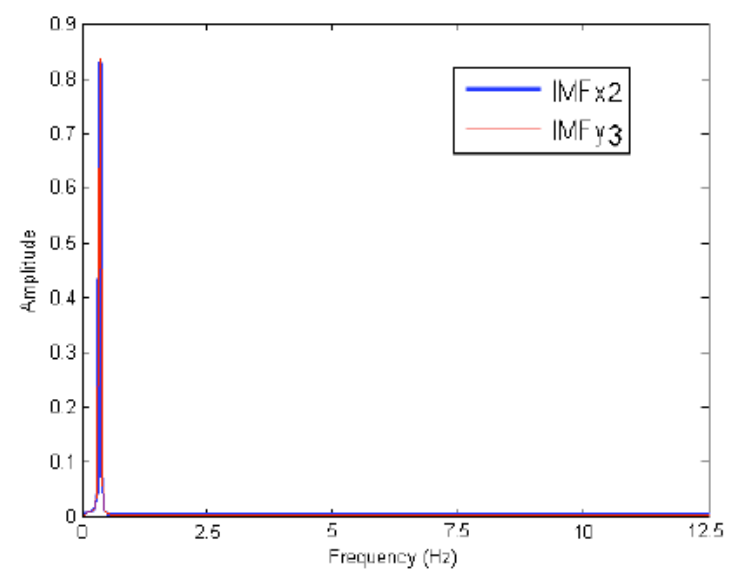

(b)

Fig. (5). (a). The PSD of $\operatorname{IMF}_{x 1}$ and $\operatorname{IMF}_{y 1}(\mathbf{b})$. The PSD of $\operatorname{IMF}_{x 2}$ and $\mathrm{IMF}_{y 3}$.

If the Cor $_{\text {Freq }}$ of two IMFs is greater than $\eta$, we consider these two IMFs are from the same 'channel'.

The $\mathrm{Cor}_{\text {Freq }}$ of IMFs between $\mathrm{IMF}_{x 1}, \mathrm{IMF}_{x 2}$ and $\mathrm{IMF}_{y 1}$, $\mathrm{IMF}_{y 2}, \mathrm{IMF}_{y 3}$ are shown in (Table 2).

Table 2. CorFreq Between IMFs

\begin{tabular}{|c|c|c|c|}
\hline & $\mathbf{I M F}$ & $\mathbf{I M F}_{\mathbf{y} \mathbf{2}}$ & $\mathbf{I M F}_{\mathbf{y} 3}$ \\
\hline \hline $\mathbf{I M F}_{\mathbf{x} 1}$ & 0.999 & -0.040 & -0.041 \\
\hline $\mathbf{I M F}_{\mathbf{x} 2}$ & -0.041 & -0.040 & 0.999 \\
\hline
\end{tabular}

From Table 2, we can easily find that $\mathrm{IMF}_{\mathrm{x} 1}$ is corresponds to $\mathrm{IMF}_{y 1}, \mathrm{IMF}_{x 2}$ is corresponds to $\mathrm{IMF}_{y 3}$ and can not find an IMF corresponds to $\mathrm{IMF}_{y 2}$ among the IMFs which decomposed from $x(t)$. Therefore, if we need to set up a oneon-one relationship between these two groups of IMFs, we should have two groups of IMFs rearranged, as shown in (Fig. 6).

In this paper, we first set up a PSD data sample series, and re-sequencing them in accordance with their power distribution in frequency domain. And then, the signal which need for identification and classification will be decomposed into IMFs, and PSDs of each IMFs will be acquired and these PSDs will have a comparison with the PSDs in the data sample by virtue of correlation coefficient. Through the use of correlation coefficient, the relationship between these IMFs are determined. After that, the IMFs which need to be analyzed are sorted in accordance with the position of their corresponding IMFs in sample series. If some of IMFs in data sample series can not be found in IMFs which decomposed from the signal been analyzed, then series of all zeros will be added into the corresponding location.

\subsection{The Selection of Feature}

The IMFs which decomposed by EMD method on behalf of a certain channel with a characteristic scales, and the amount of energy go through the channel can characterize the operation of the machine. Therefore, we select the energy of each IMFs as the feature parameters. We define the energy of a IMF as $E_{\mathrm{IMF} i}$, and the total energy as $E$.

$$
\begin{aligned}
& E_{\mathrm{IMF}_{i}}=\int_{-\infty}^{\infty} \mathrm{IMF}_{i}^{2}(t) d t \\
& E=\sum_{i=1}^{n} E_{I M F_{i}}
\end{aligned}
$$

Considering that the energy values are often large, in order to facilitate follow-up analysis and calculation, we make these energy value normalized. 

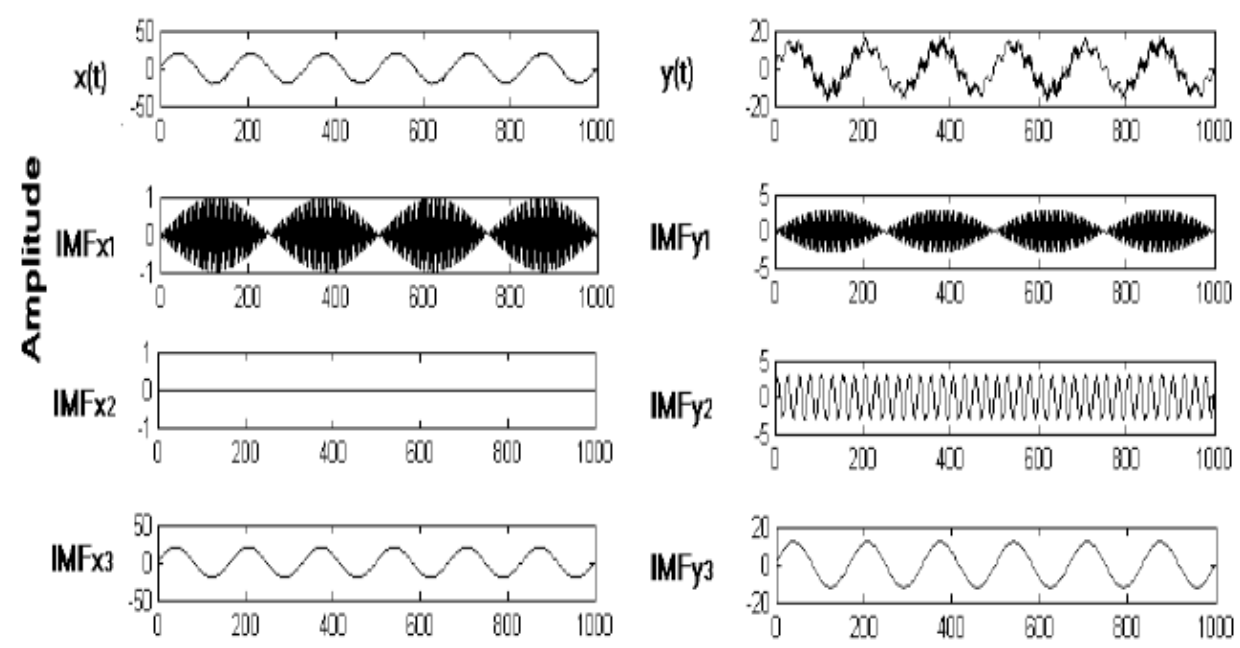

IMFy

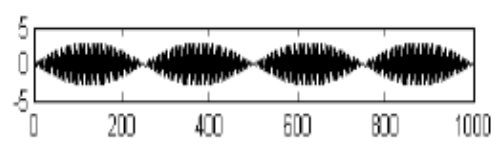

IMFy2

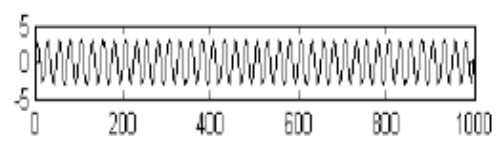

IMFy3

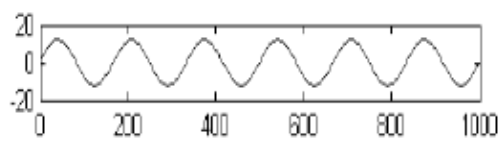

Fig. (6). Rearranged IMFs.

$$
\bar{E}_{I M F_{i}}=\frac{E_{I M F_{i}}}{E}
$$

$\overline{\mathrm{E}}_{\mathrm{IMF} i}$ is the normalized feature parameters.

After these feature parameters have been obtained, the SVM technology can be used to classify and assess these parameters. The operation procedure of the whole intelligent fault diagnosis is shown in (Fig. 7).

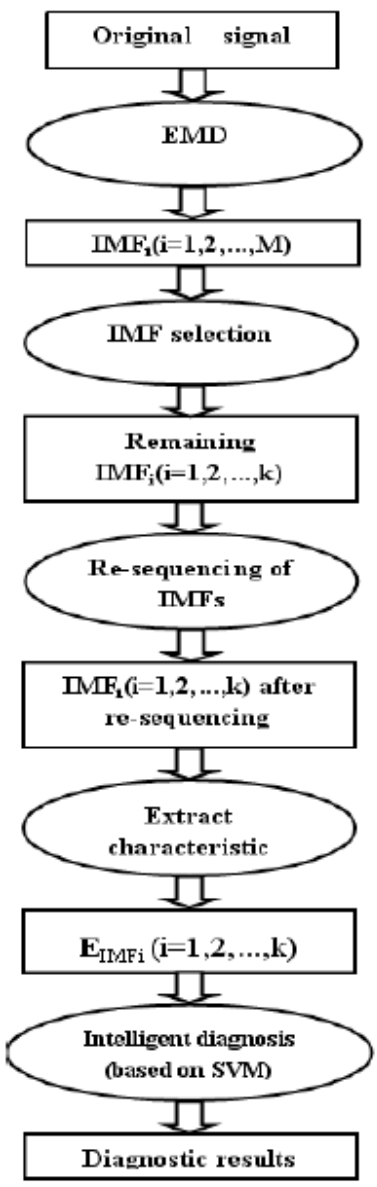

Fig. (7). The operation procedure of intelligent fault diagnosis.

\section{EXPERIMENT AND DATA SET}

\subsection{Experiment}

In order to validate the proposed method, a experiment was carried out. The data of two types of fault status and normal status were obtained from a test rig. The test rig shown in (Fig. 8) is used for modeling different fault types such as local rub-impact, imbalance and misalignment fault.

Vibration data was collected using eddy current displacement sensor,which was attached to the foundation with magnetic base. Eddy current displacement sensor was placed close to the disk. Vibration signals were collected using a data acquisition card with sample rate $3 \mathrm{~K} / \mathrm{s}$. The experimental rotating speed is about $1500 \mathrm{rpm}$.

As shown in Fig. (8), the test rig is driven by a DC motor. In order to reduce the influence of motor's vibration on shaft, the coupling between the motor and main shaft is flexible.

As mentioned earlier in this paper, data of three different status were measured, there are normal status, imbalance fault status and rub-impact fault status. There are totally 360 samples were obtained, each status have 120 samples and each sample in the data sets includes 1000 points.

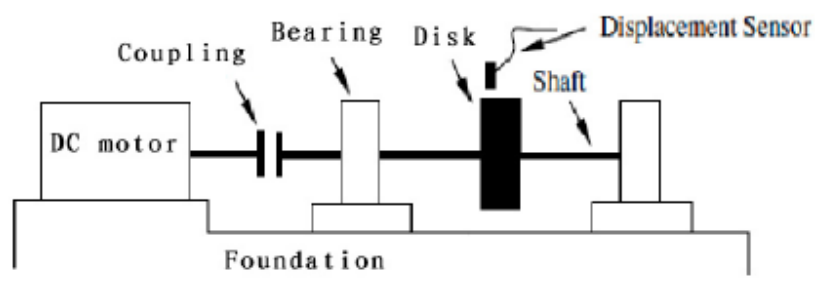

Fig. (8). Test rig.

\subsection{The Selection and Re-Sequence of IMFs}

Fig. (9) is the EMD decomposition result of three different signal. All the IMFs of vibrations signals from the same testing rig at the same speed with normal, imbalance fault and rub-impact fault have been shown in Fig. (9a), (9b) and (9c) respectively. 

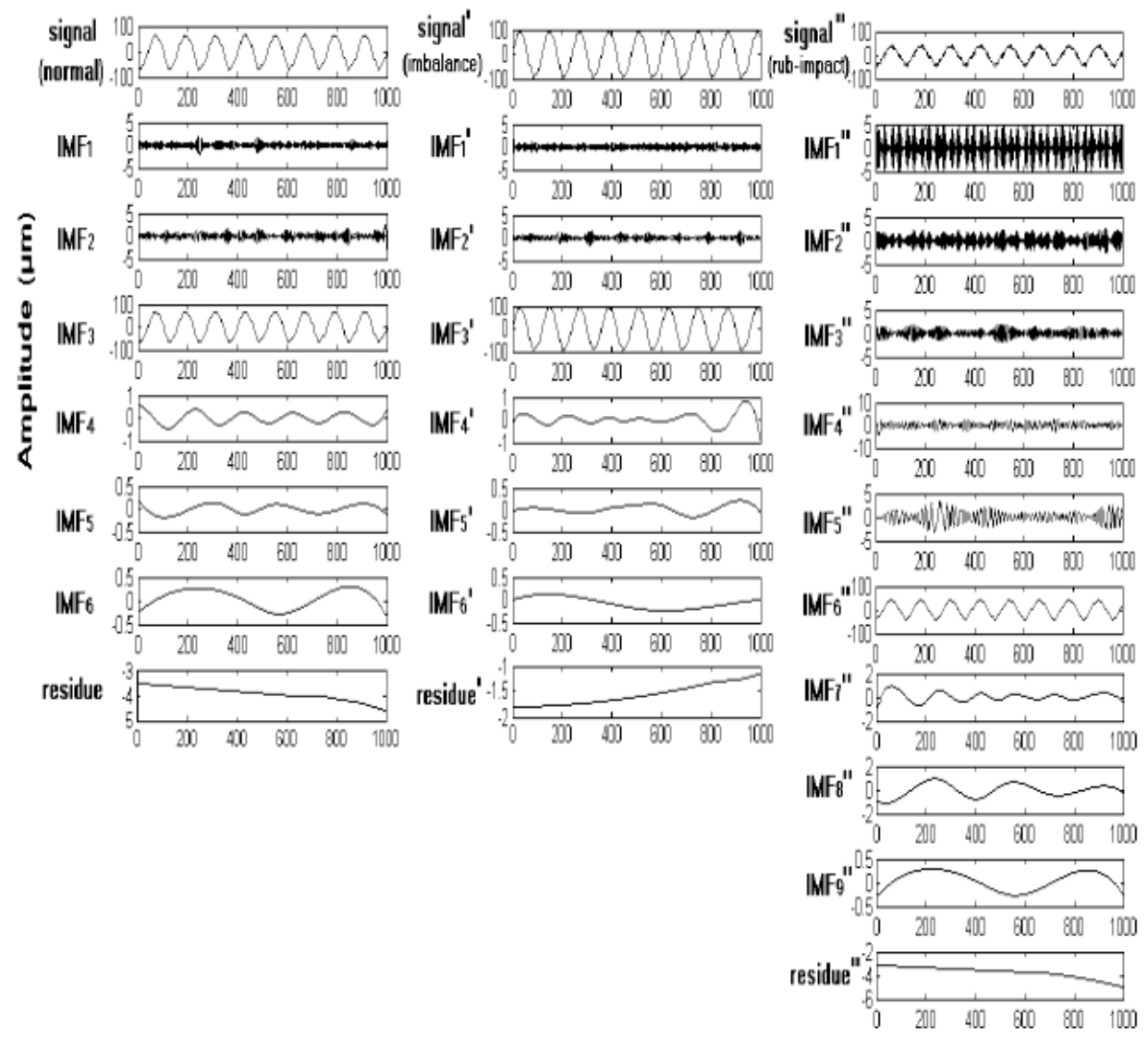

(a)

(b)

(c)

Fig. (9). IMFs of three different signals:(a) normal status (b) imbalance falut (c) rub-impact fault.

After IMF selection with IMF selection criterion, only the first three IMFs of $\mathrm{IMF}_{i}$ (decomposed from signal in normal status) and $\mathrm{IMF}_{i}^{\prime}$ (decomposed from signal in imbalance status) are retained, and when it comes to the analysis of the signal with rub-impact fault only the first six IMFs of $\mathrm{IMF}_{i}{ }^{\prime \prime}$ are retained, the others are eliminated. The retained IMFs are shown in (Fig. 10).

Because the signal"(rub-impact) decomposed of the largest number of IMFs (there are six IMFs decomposed from signal", we define them as $\mathrm{IMF}_{i}{ }^{\prime \prime}$ ), we set $\mathrm{IMF}_{i}{ }^{\prime \prime}$ as the sample series. Calculate the $\mathrm{Cor}_{\text {Freq }}$ between $\mathrm{IMF}_{\mathrm{i}}$ (which decomposed from signal in normal status) and $\mathrm{IMF}_{i}{ }^{\prime \prime}, \mathrm{Cor}_{\text {Freq }}$ between $\mathrm{IMF}_{i}$ ' (which decomposed from signal in imbalance status) and $\mathrm{IMF}_{i} "$ are also calculated correspondingly. The calculation result were shown in (Table 3 and Table 4), and the largest frequency domain correlation coefficient of each IMFs is denoted in bold face.

From Table 3 and Table 4, we can find that $\mathrm{IMF}_{1}$ and $\mathrm{IMF}_{1}{ }^{\prime}$ have the largest $\mathrm{Cor}_{\text {Freq }}$ with $\mathrm{IMF}_{2}{ }^{\prime \prime}, \mathrm{IMF}_{2}$ and $\mathrm{IMF}_{2}{ }^{\prime}$ have the largest $\mathrm{Cor}_{\text {Freq }}$ with $\mathrm{IMF}_{3}{ }^{\prime \prime}, \mathrm{IMF}_{3}$ and $\mathrm{IMF}_{3}$ ' have the largest $\mathrm{Cor}_{\text {Freq }}$ with $\mathrm{IMF}_{6} "$.

We can find from the distribution of PSD that these IMFs have very similar distribution of power on the frequency domain, as shown in (Fig. 11, Fig. 12 and Fig. 13).

In this way, we set up a one-on-one relationship between these groups of IMFs, and then acquired the three groups of IMFs by re-sequencing these IMFs, as shown in (Fig. 14).

\subsection{Classification Performance of the Energy}

In this paper, we use energy as the feature to characterization of each IMFs. Energy value is essentially a measure of how many energy go through a particular frequency channel. When the condition of mechanical equipment changed, the energy of each IMFs will be different from the normal condition. Thus the energy contains the fault information and can be used as a feature for diagnosis. In order to evaluate the classification performance of the energy, the SVMs are used to train and test on these three data sets, corresponds to normal status, imbalance fault and rub-impact fault. For the above three kinds of status, there are 72 study samples for each status.

We adopt "one-against-one" method to identify the different faults. Radial basis kernel function was chosen as kernel function.Finding optimal model parameters by discrete grid search in parameter space $\left[2^{-5}, 2^{-10}\right]$.

After the study, there are 144 test samples ( each status has 48 test samples) for SVMs to test the classification accuracy rate of SVMs. Test result show that 137 samples have been correctly classified and 7 samples have been wrongly classified, the validation classification accuracy rate of SVMs is $95.14 \%,(\mathrm{C}, \gamma)$ represents the optimal parameters of SVMs, the $(C, \gamma)=\left(2^{10}, 2^{3}\right)$ in this test. The classification accuracy rate implies that the method proposed in this paper can correctly discriminate the condition of machinery. 


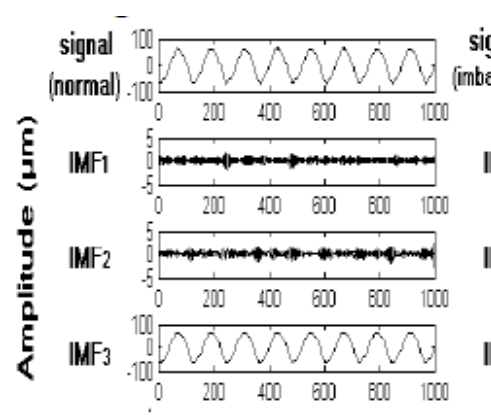

(a)

(b)

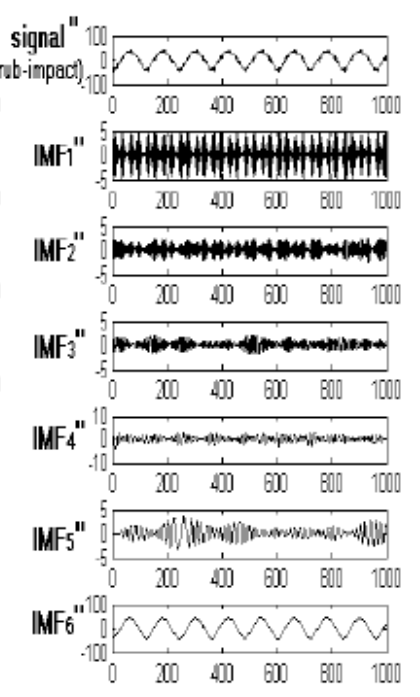

Fig. (10). The retained IMFs after selection:(a) normal status (b) imbalance falut (c) rub-impact fault.

Table 3. The Frequency Domain Correlation Coefficient Between $\operatorname{IMF}_{i}$ and $\operatorname{IMF}_{i}{ }^{\prime \prime}$

\begin{tabular}{|c|c|c|c|c|c|c|}
\hline & $\mathbf{I M F}_{1}{ }^{\prime \prime}$ & $\mathbf{I M F}_{2}{ }^{\prime \prime}$ & $\mathbf{I M F}_{3}{ }^{\prime \prime}$ & $\mathbf{I M F}_{4}{ }^{\prime \prime}$ & $\mathbf{I M F}_{\mathbf{5}}{ }^{\prime \prime}$ & IMF $_{\mathbf{6}}{ }^{\prime}$ \\
\hline \hline $\mathbf{I M F}_{\mathbf{1}}$ & -0.098 & $\mathbf{0 . 6 9 4}$ & 0.148 & -0.068 & -0.154 & -0.025 \\
\hline $\mathbf{I M F}_{2}$ & -0.149 & 0.107 & $\mathbf{0 . 7 8 3}$ & 0.225 & -0.027 & -0.014 \\
\hline $\mathbf{I M F}_{3}$ & -0.015 & -0.032 & -0.022 & -0.011 & -0.007 & $\mathbf{0 . 8 6 6}$ \\
\hline
\end{tabular}

Table 4. The Frequency Domain Correlation Coefficient Between $\operatorname{IMF}_{i}$ ' and $\operatorname{IMF}_{i}{ }^{\prime \prime}$

\begin{tabular}{|c|c|c|c|c|c|c|}
\hline & IMF $_{1}$ & $\mathrm{IMF}_{2} "$ & IMF $_{3} "$ & $\mathrm{IMF}_{4} "$ & IMF $_{5} "$ & $\mathrm{IMF}_{6} "$ \\
\hline IMF1' & -0.056 & 0.650 & -0.097 & -0.192 & -0.179 & -0.016 \\
\hline IMF2' & -0.144 & 0.265 & 0.694 & -0.018 & -0.137 & -0.014 \\
\hline IMF3' & -0.011 & -0.025 & -0.017 & -0.008 & -0.006 & 0.993 \\
\hline
\end{tabular}

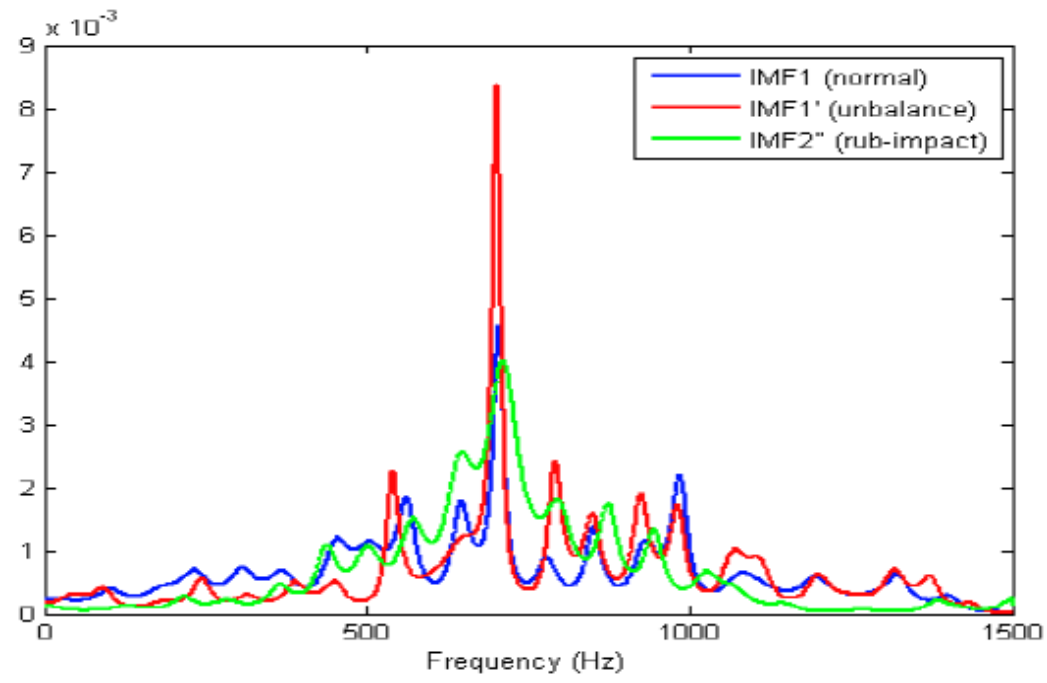

Fig. (11). PSD of IMF1, IMF1' and IMF2". 


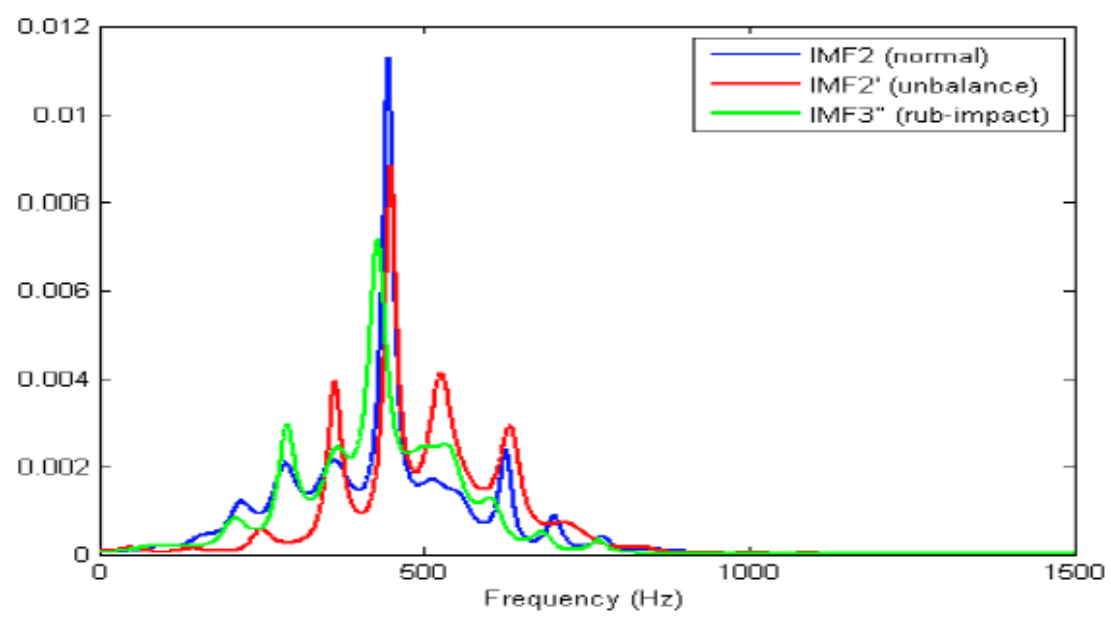

Fig. (12). PSD of IMF2, IMF2' and IMF3".

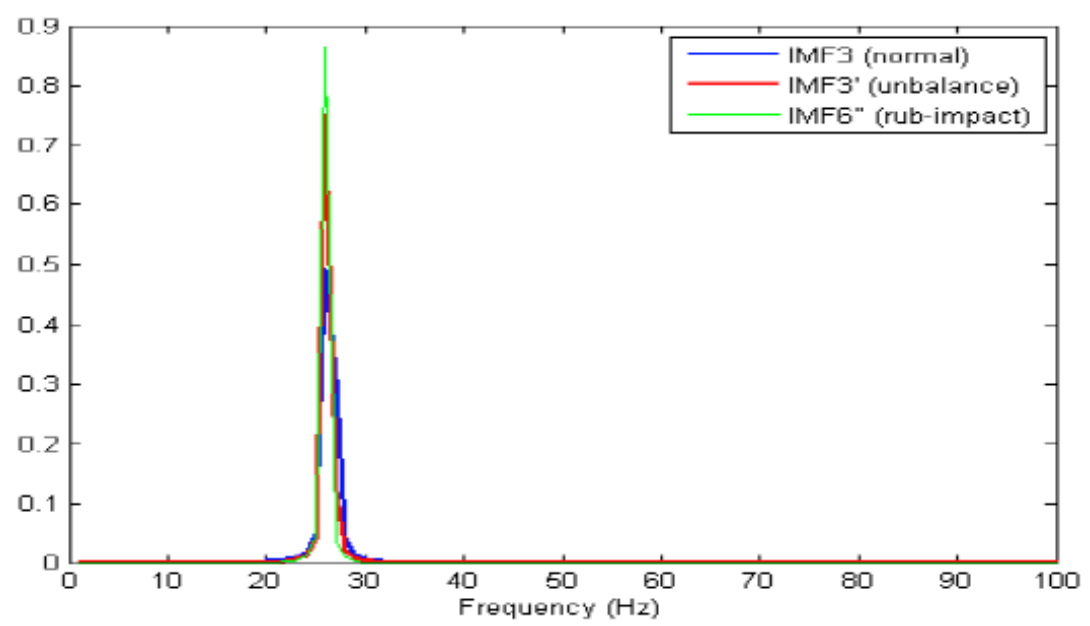

Fig. (13). PSD of IMF3, IMF3' and IMF6".

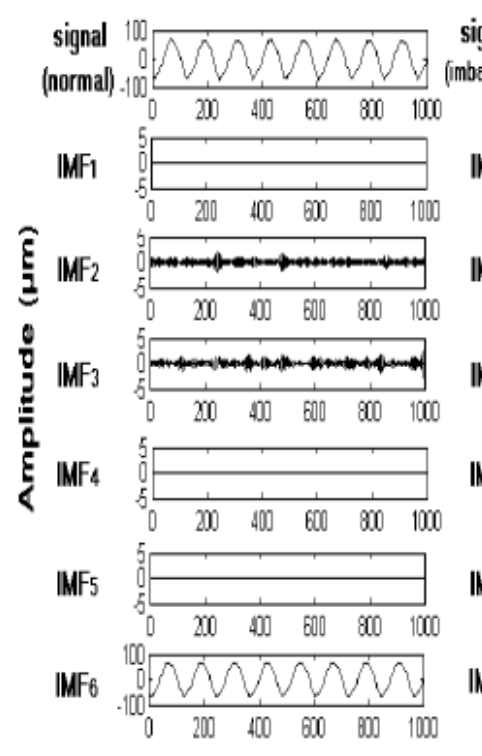

(a)

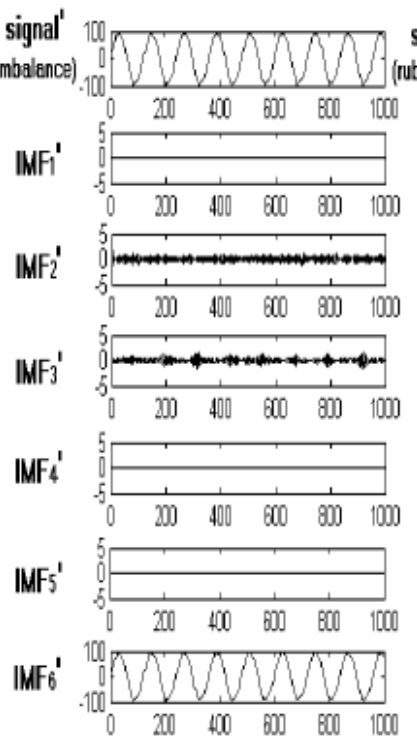

(b)

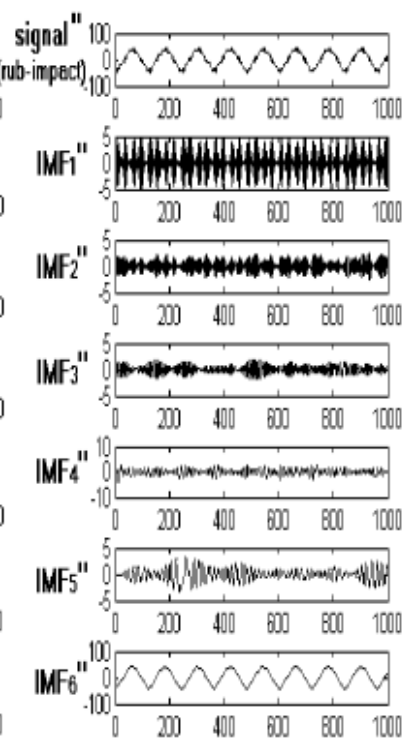

(c)

Fig. (14). Rearranged IMFs: (a) normal status (b) imbalance falut (c) rub-impact fault. 


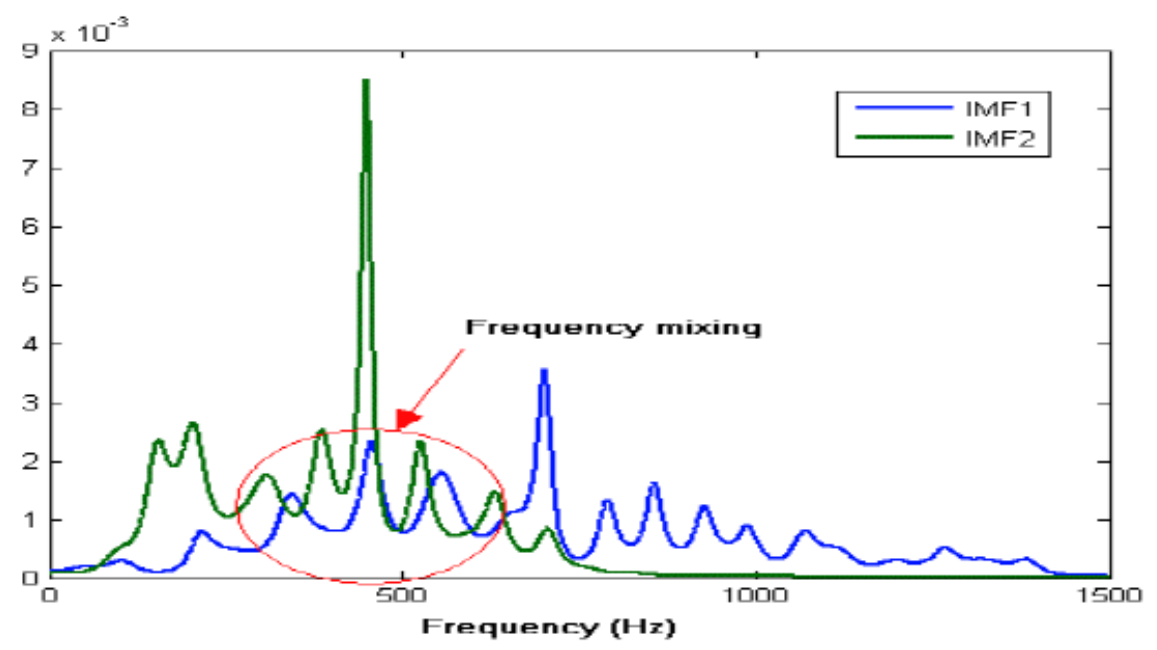

Fig. (15). Frequency mixing.

\subsection{Result Analysis}

By analyzing the signal which were wrongly classified, we find that the main causes of classification error is due to the frequency mixing problem. That is to say, in some case, adjacent IMFs will contain the same frequency components in a EMD decomposition. Usually, EMD decomposition can effectively decompose different frequency component into different IMFs, frequency mixing will be under effective control. But sometimes the degree of frequency mixing can be very serious, as shown in (Fig. 15). We can see from the PSD that the two IMFs all have a large power density distribution in the region marked with a red circle. This leads to larger error of energy value, eventually causing classification errors.

\section{DISCUSSION}

(1) Through the analysis of simulation data and experiment data, it can be seen that EMD is a powerful tool for fault diagnosis in rotating machinery. And by using correlation coefficient, we set a one-on-one relationship between different IMFs. This made it feasible for the intelligent fault diagnosis based on EMD method.

(2) Although we verified the feasibility and effectiveness of the intelligent fault diagnosis based on the EMD method, this method still need to be improved in many fronts. Especially the phenomenon of frequency mixing., which seriously reduced the accuracy of classification. How to make each IMF has a more specific physical meaning is still a problem need to be solve. The authors would like to investigate this topic in future.

\section{CONCLUSIONS}

The empirical mode decomposition (EMD) method provides a powerful tool for nonlinear and non-stationary signal analysis. However, EMD method does not suitable for intelligent fault diagnosis due to some characteristics of its own. To overcome this shortcoming, we propose a new method based on correlation coefficient to set up a one-on-one relationship between these IMFs which decomposed from different signal. In this paper, three kinds of vibration signals from a test rig are analyzed using the proposed method to diagnose the faults. The application results show that the proposed method is able to correctly discriminate the condition of machinery.

\section{CONFLICT OF INTEREST}

The author(s) confirm that this article content has no conflicts of interest.

\section{ACKNOWLEDGEMENT}

This work is partly supported by the science and technology major project of China (No. 2012ZX04012031).

\section{REFERENCES}

[1] P. Flandrin, G. Rilling and P. Goncalvès, "Empirical mode decomposition as a filter bank", IEEE Signal Processing Letter, vol. 11, no. 2, pp. 112-114, 2004.

[2] V.K. Rai and A.R. Mohanty, "Bearing fault diagnosis using FFT of intrinsic mode functions in Hilbert-Huang transform", Mechanical Systems and Signal Processing, vol. 21, no. 6, pp. 2607-2615, 2007.

[3] Y.G. Lei, Z.J. He and Y.Y. Zi, "Fault diagnosis of rotating machinery based on multiple ANFIS combination with Gas", Mechanical Systems and Signal Processing, vol. 21, no. 5, pp. 2280-2294, 2007.

[4] Q.H. Du and S.N. Yang, "Application of the EMD method in the vibration analysis of ball bearings", Mechanical Systems and Signal Processing, vol. 21, no. 5, pp. 2634-2644, 2007.

[5] J.S. Cheng, D.J. Yu, J.S. Tang and Y. Yang, "Application of frequency family separation method based upon EMD and local Hilbert energy spectrum method to gear fault diagnosis", Mechanism and Machine Theory, vol. 43, no. 6, pp. 712-723, 2008.

[6] J.S. Cheng, D.J. Yu, J.S. Tang and Y. Yang, "Local rub-impact fault diagnosis of the rotor systems based on EMD", Mechanism and Machine Theory, vol. 44, no. 6, pp. 784-791, 2008.

[7] Q. Gao, C. Duan, H. Fanc and Q. Meng, "Rotating machine fault diagnosis using empirical mode decomposition", Mechanical Systems and Signal Processing, vol. 22, no. 5, pp. 1072-1081, 2008.

[8] Y. Lei, "Application of the EEMD method to rotor fault diagnosis of rotating machinery", Mechanical Systems and Signal Processing, vol. 23, no. 4, pp. 1327-1338, 2009.

[9] Z.K. Penga, Peter W. Tsea and F.L. Chu, "A comparison study of improved Hilbert-Huang transform and wavelet transform: Application to fault diagnosis for rolling bearing", Mechanical Systems and Signal Processing, vol. 19, no. 5, pp. 974-988, 2005. 
[10] Y. Maki and K.A. Loparo, "A neural network approach to fault detection and diagnosis in industrial process", IEEE Transactions on Control System Technology, vol. 5, no. 6, pp. 529-541, 1997.

[11] B.A. Paya, I.I. Esat and M.N.M. Badi, "Artificial neural network based fault diagnostics of rotating machinery using wavelet transforms as a preprocessor", Mechanical Systems and Signal Processing, vol. 11, no. 5, pp. 751-765, 1997.

[12] Y.S. Zhu and Y.Y. Zhang, "Support vector machine and its application in mechanical fault pattern recognition", Ph.D. Thesis, Department of Mechanical Engineer, Xi'an Jiaotong University, Xi'an, China, 2003.

[13] N.E. Huang, Z. Shen and S.R. Long, "The Empirical mode decomposition and the Hilbert spectrum for nonlinear and non-stationary time series analysis", Proceedings of the Royal Society of London Series A, vol. 454, pp. 903-995, 1998.
[14] V.N. Vapnik, "The nature of statistical learning theory", New York, Springer, 1995.

[15] J.Y. Yang, Y.Y. Zhang and Y.S. Zhu, "Intelligent fault diagnosis of rolling element bearing based on SVMs and fractal dimension", Mechanical Systems and Signal Processing, vol. 21, no. 5, pp. 2012-2024, 2007.

[16] C.W. Hsu and C.J. Lin, "A comparison of methods for multi-class support vector machines", IEEE Transactions on Neural Networks, vol. 13 , no. 2,2002 , pp. 415-425.

[17] J.P. Burg, "Maximum entropy spectral analysis", PhD Thesis, Stanford University, 1975.

[18] Y.Q. Xu, S.Y. Deng, X.W. Li and X.K. Chen, "Application of comparison method with correlation coefficient array in identification of chinese herbs", Spectroscopy and Spectral Analysis, vol. 11, no. 7, pp. 2239-2242, 2007.

Received: August 13, 2013

Revised: August 28, 2013

Accepted: August 28, 2013

(C) Zhang et al.; Licensee Bentham Open.

This is an open access article licensed under the terms of the Creative Commons Attribution Non-Commercial License (http://creativecommons.org/licenses/by-nc/3.0/) which permits unrestricted, non-commercial use, distribution and reproduction in any medium, provided the work is properly cited. 